\title{
The Effect of Salvianolic Acid on Vascular Protection and Possible Mechanisms
}

\author{
Yalan $\mathrm{Wu},{ }^{1}$ Suowen $\mathrm{Xu},{ }^{2}$ and Xiao Yu Tian ${ }^{1}{ }^{1}$ \\ ${ }^{1}$ School of Biomedical Sciences, Institute of Vascular Medicine, Chinese University of Hong Kong, Shatin, N.T. Hong Kong SAR, China \\ ${ }^{2}$ Department of Endocrinology, First Affiliated Hospital, Division of Life Sciences and Medicine, University of Science and Technology \\ of China (USTC), Hefei, China
}

Correspondence should be addressed to Xiao Yu Tian; xytian@cuhk.edu.hk

Received 15 July 2020; Revised 24 August 2020; Accepted 1 September 2020; Published 25 September 2020

Academic Editor: Marcos R. de Oliveira

Copyright (c) 2020 Yalan Wu et al. This is an open access article distributed under the Creative Commons Attribution License, which permits unrestricted use, distribution, and reproduction in any medium, provided the original work is properly cited.

Salvia miltiorrhiza (Danshen), as an important traditional Chinese medicinal plant, has been used in China for the treatment of cardiovascular diseases for hundreds of years. Salvianolic acids (salvianolic acid A and salvianolic acid B) as the most abundant water-soluble component extracted from Salvia miltiorrhiza have attracted more and more attention from cardiovascular scientists due to its comprehensive cardiovascular actions. In vivo and in vitro studies have rendered salvianolic acid an excellent drug candidate for the treatment and prevention of cardiovascular diseases. In this review, we surveyed the protective effects of salvianolic acid A and salvianolic acid B against cardiovascular diseases and the pharmacological basis, providing a strong scientific rationale for elucidating the important role of Salvia miltiorrhiza in cardiovascular therapy. More importantly, we also hope to provide new inspiration and perspectives on the development and innovation of small-molecule cardiovascular drugs based on salvianolic acid.

\section{Introduction}

Cardiovascular disease, as one of the major noncommunicable chronic diseases that threaten human health and affect quality of life around the globe, has increasing incidence nowadays, with high rates of disability and mortality rate [1-3]. Therefore, searching for effective drugs to intervene cardiovascular diseases has become a top priority for researchers.

Chinese herbal medicine is a valuable resource to identify new small-molecule drugs against human disease. Empirical evidences from the application of either single or multiple medicinal products (including plants, minerals, or animal products) against cardiovascular disease, by Chinese medicine (TCM) practitioners, provide a lot of insights to identify potential bioactive extracts [4]. In recent years, Chinese TCM practitioners and clinicians have also integrated TCM with modern medicine, resulting in substantial improvement to dealing with cardiovascular and cerebrovascular diseases, with significantly reduced mortality and much better quality of life [4]. One of the most successful examples is the identification of several herbs or herbal extracts, as effective drugs to treat cardiovascular disease, which "improves blood circulation," based on the TCM theory and practice to treatment of "blood stasis." Among them include the most commonly used TCM herbs, such as Danshen (Salvia miltiorrhiza, also called red sage), Chuanxiong (Ligusticum striatum, also called Szechuan lovage), and Baishao (Paeonia lactiflora, also called white peony), which have been studied most extensively in practice, resulting in the discovery of several small molecules including salvianolic acid from Danshen [5].

Danshen belongs to the TCM herb category of promoting blood circulation and removing blood stasis. In TCM practice, it has been applied usually in combination with other herbs for thousands of years in Asian countries to treat vascular disorder-related diseases, including coronary heart disease, myocardial infarction (MI), angina pectoris, and atherosclerosis [5-8]. The effectiveness and wide usage of Danshen have led to the development of a compound Danshen dripping pill, or Dantonic ${ }^{\circledR}$, a major innovation in TCM. Dantonic ${ }^{\circledR}$, which has been used since the 1990s, is also the first compound TCM drug to pass the second phase of human clinical trial of the US Food and Drug Administration 
(FDA). A meta-analysis of 60 randomized clinical trials showed that Danshen dripping pills composed of Salvia miltiorrhiza, Panax notoginseng, and camphor have a more obvious effect than isosorbide dinitrate $[5,9,10]$.

So far, several chemical components of Danshen have been identified, including more than 30 lipophilic compounds with a diterpene structure, such as tanshinones IVI, cryptotanshinone, isothiophenes I-II, and salvianphenol $\mathrm{A}$, and more than 50 hydrophilic compounds, such as danshensu, salvianolic acid A (SalA), salvianolic acid B (SalB), and protocatechuic aldehyde, mostly composed of phenolic acids $[5,9,10]$ (Figure 1). The contents of chemicals in S. miltiorrhiza roots vary among aqueous extracts, organic extracts, oral pills, and injections derived from the single herb of S. miltiorrhiza roots or herbal combination, like Fufang (multiple TCM herbs) prescription in China. Salvianolic acids are the most abundant water-soluble substances in Danshen. Many in vivo and in vitro studies have shown that salvianolic acid regulates multiple signal transduction pathways in vascular endothelial cells, vascular smooth muscle cells, and cardiomyocytes. In this review, we systematically surveyed the research progress of the protective effect of salvianolic acids (both SalA and SalB) on blood vessels and vascular cells, with a focus on its regulation of vascular endothelium and smooth muscle and signaling pathways related to oxidative stress, aiming to provide a scientific basis for understanding the traditional use of Salvia miltiorrhiza in vascular protection.

\section{Evidence-Based Therapeutic Applications of Sal-Containing TCM Drugs}

One of the most commonly used TCM drug Dantonic (or Fufang Danshen) has been recorded in the "Chinese Pharmacopoeia" (2015) which documented its application in conditions such as coronary atherosclerosis, angina pectoris, hyperlipidemia, and Alzheimer's disease in China and some other Asian countries. It is a China Food and Drug Administration- (CFDA-) approved drug since 2008. Dantonic and other forms of Fufang Danshen contain S. miltiorrhiza, Panax notoginseng, and Ligusticum wallichii, from which the composition of these drugs is originally based on TCM theory of allowing the three herbs to synergize and thus exert the desirable effects on treating cardiovascular disease and at the same time to antagonize the side effects of each other. This formulation contains many active ingredients including tanshinone, salvianolic acid, Panax notoginseng saponins, ginsenosides Rb1 and Rg1, and borneol. Many studies have proved its effectiveness, including relieving pain, promoting blood circulation, improving blood flow, reducing blood lipids, protecting blood vessels and myocardium, and improving cardiac function.

The clinical effect of S. miltiorrhiza, or its active ingredient tanshinone IIA, or Fufang Danshen, has been examined in patients with various conditions of cardiovascular diseases in several clinical studies from about 1980s. However, due to the limited information available only for literature written in Chinese, these evidences are not discussed here, although, collectively, they suggested the effectiveness of S. miltiorrhiza<smiles>O=C(/C=C/c1ccc(O)c(O)c1/C=C/c1ccc(O)c(O)c1)OC(Cc1ccc(O)c(O)c1)C(=O)O</smiles><smiles>CC(C)(C)CCCCCCC(=O)OC(Cc1ccc(O)c(O)c1)C(=O)O</smiles>

Figure 1: Chemical structures of salvianolic acid A (SalA) and salvianolic acid B (SalB).

in coronary heart disease, stroke, thrombosis, etc. A later study showed that compared with routine care as a control, S. miltiorrhiza preparation plus routine care in patients with acute myocardial infarction reduced the mortality rate by approximately half [11]. However, many studies examined either Fufang Danshen, the extract of S. miltiorrhiza, or the compound tanshinone IIA, whereas the effects of SalA and SalB were much less explored in human subjects. For example, one study showed that Fufang Danshen reduced systolic blood pressure compared to placebo in patients with hypertension [12]. Another study showed that S. miltiorrhiza improved the prognosis of patients after percutaneous coronary intervention [13]. A meta-analysis in 2016 summarized data from over 2000 patients and concluded that S. miltiorrhiza was effective for unstable angina pectoris [14]. A later study demonstrated that $\mathrm{S}$. miltiorrhiza injection reduces oxidative stress during the percutaneous coronary intervention perioperative period, increases myocardial perfusion, and promotes the recovery of cardiac function [15]. Despite all these clinical evidences, whether SalA or SalB only has effect which is similar to the total extract of S. miltiorrhiza in treating cardiovascular disease in humans is still unclear.

\section{Protective Effects of Salvianolic Acids A and $B$ against Oxidative Stress}

Numerous studies have shown that different types of polyphenols have a wide range of pharmacological activities in endothelial cells and cardiomyocytes $[16,17]$. The effect of SalA to inhibit oxidative stress implicates its potential to treat many conditions associated with excessive oxidative stress, including endothelial dysfunction, pulmonary arterial hypertension, and cardiac fibrosis [18-23], mainly through a direct effect of SalA to scavenge various types of free radicals. For 
example, SalA can chelate $\mathrm{Cu}^{2+}$ and inhibit $\mathrm{Cu}^{2+}$-mediated oxidation of low-density lipoprotein, reducing the production of malondialdehyde (MDA), the end product of lipid oxidation in a cell-free system [24]. The protective effects of SalA on low-density lipoprotein (LDL) oxidation implicate its potential effects on the formation of oxidized LDL (oxLDL) and consequent oxLDL-mediated proatherogenic events in the pathogenesis of atherosclerosis.

\subsection{Direct Effect of SalA and SalB to Scavenge Free Radicals.} As a polyphenol, SalA has a direct free radical scavenging effect [25]. Apart from the effect on lipid peroxidation [24], both SalB and SalA also show direct free radical scavenging ability in a cell-free system [26, 27]. Liu et al. reported that seven phenolic compounds isolated from Danshen inhibited lipid peroxidation of rat liver microsomes induced by iron/ cysteine and vitamin $\mathrm{C} / \mathrm{NADPH}$ [28]. These compounds also inhibit $\mathrm{H}_{2} \mathrm{O}_{2}{ }^{-}$-induced hemolysis of rat red blood cells in vitro [28]. SalA is generally considered a more effective antioxidant than SalB, although SalB is more abundant in Danshen [27-29]. The scavenging activity of SalB is higher than vitamin $\mathrm{C}$ on scavenging $\mathrm{HO}, \mathrm{O}_{2}{ }^{--}$, DPPH free radicals, and ABTS free radicals, whereas its effect to chelate iron or scavenge $\mathrm{H}_{2} \mathrm{O}_{2}$ is lower than that of vitamin $\mathrm{C}$ [26]. It is reported that treatment with $5 \%$ water-soluble extract of Danshen which contained SalB for 12 weeks lowers blood cholesterol and reduces atherosclerotic plaque formation in diet-induced hypercholesterolemic rabbits, which is associated with its reactive oxygen species (ROS) scavenging capacity [30]. Moreover, SalB-treated LDL has vitamin E binding capacity and is resistant to $\mathrm{Cu}^{2+}$-induced oxidation [30]. Intravenous injection of SalA in a range of $0.3-3 \mathrm{mg} / \mathrm{kg}$ significantly attenuates isoproterenol-induced cardiac dysfunction and myocardial injury and improves mitochondrial respiratory function in rats with isoproterenol-induced myocardial infarction [31], which is also associated with the antioxidant capacity of SalA.

3.2. Modulation of Oxidative and Antioxidative Mechanisms and Molecules by SalA and SalB. SalA also exert antioxidative effects through enhancing the antioxidative arm or inhibiting oxidative enzymes in the vascular cells. For instance, SalA downregulates the expression of NADPH oxidase 4 (NOX4) [32], which produces reactive oxygen species in the disease model of pulmonary fibrosis. In addition, SalA can also upregulate the Nrf2/heme oxygenase-1 (HO-1) signaling pathway to exert its antioxidant effects [33-35]. Oxidative stress is a critical player during the process of endothelialmesenchymal transition (EndMT) [26,36], a cellular process important for vascular remodeling changes. Chen et al. previously reported the effect of SalA during EndMT of human pulmonary artery endothelial cells (HPAECs), as well as in vivo on pulmonary vascular remodeling induced by monocrotaline in rats [37]. EndMT is induced by TGF $\beta 1$ in human pulmonary artery endothelial cells (HPAECs). SalA attenuates EndMT in HPAECs induced by TGF $\beta 1$, through inhibition of cell migration and reactive oxygen species (ROS) production. In the monocrotaline-induced pulmonary hypertension (PAH) model, SalA improves vascular function, decreases TGF $\beta 1$ expression, and inhibits inflammation. They also showed that these effects are attributed to SalAinduced Nrf2 translocation and subsequent upregulation of HO-1 [37]. Targeting reactive oxygen species (ROS) through Nrf2 and HO-1 seems to be one of the major downstream effectors for the effect of SalA in the vasculature.

Excessive ROS production enhances the proliferation of aortic vascular smooth muscle cells (VSMCs). Hung et al. showed that SalA and SalB inhibit the proliferation of rat aortic VSMC cell line A10 which is stimulated by homocysteine (a cardiovascular risk factor known to trigger ROS). By using proteomics, they further showed that Salvia miltiorrhiza root aqueous extract containing 3,4-dihydroxybenzoic acid, 3,4dihydroxyphenyl lactic acid, and salvianolic acid B inhibits the activation of protein kinase $\mathrm{C}$ and $\mathrm{p} 44 / 42$ MAP kinase to reduce ROS induced by homocysteine in VSMCs [38]. Interestingly, the aqueous extract also reduces carbonylation (a protein modification induced by oxidative stress) of several cytoskeletal and chaperone proteins (such as vimentin, a4 tropomyosin, and GRP75) in VSMCs [38]. ROS scavenging activity of salvianolic acid also mediates its protective effect on cardiomyocytes against doxorubicin-induced cardiotoxicity, through SalA-induced transition of $\mathrm{HO}^{\prime}$ produced by electron transfer on doxorubicin cysteine free radicals to $\mathrm{H}_{2} \mathrm{O}_{2}$ as the end product [39]. Treatment with salvianolic acid, containing mostly SalB (64.92\%), has similar effect against doxorubicin-induced cardiotoxicity by inhibiting oxidative stress [40]. These studies suggest that SalA and SalB have potent antioxidant effects either by direct scavenging or by modulating expression of oxidant/antioxidant enzymes and upstream regulators including protein kinases.

Similar to its effect of directly reacting with free radicals, SalA and SalB also directly interact with phosphoproteins, many of which are important players in signal transduction. For instance, Liu et al. studied the putative protein targets of salvianolic acid [41] and showed that salvianolic acid binds to many proteins including an important vascular extracellular matrix protein matrix metalloproteinase MMP-9 [42], as well as some other protein targets including extracellular-signalregulated kinase 1/2 (ERK1/2), c-Jun $\mathrm{NH}(2)$-terminal kinase (JNK), and p38 mitogen-activated protein kinase pathway [43-48], most likely through the interaction of SalA and SalB with the phosphotyrosine or phosphoserine/threonine binding domains $[49,50]$. Using a similar approach, Sperl et al. also reported that SalA and SalB are inhibitors of proteinprotein interactions at the $\mathrm{SH} 2$ domain of the Src family kinases Src and Lck. The potency of SalA and SalB (from 0 to $100 \mu \mathrm{mol} / \mathrm{L}$ ) in combination with Src and Lck is higher than that of rosmarinic acid, a known Lck SH2 domain inhibitor [51]. Since Lck is a T cell-restricted Src family protein tyrosine kinase and is critical in the TCR-mediated signaling pathway, the activity of Lck SH2 domain makes SalA and SalB optimal candidates for immune-suppressive anticancer agents, similar to rosmarinic acid $[52,53]$. A recent report by Wang et al. used an ELISA-like high-throughput screening assay to show that SalB and rosmarinic acid have high affinity to bind and to inhibit $\mathrm{SH} 2$ domain of CD36, a membrane receptor of oxLDL, thus preventing oxLDL from being taken up by macrophages [54]. Since both SalA and 
SalB have a similar core structure of rosmarinic acid [51], their high affinity for the Src and the SH2 domain of CD36 indicates the role of immunomodulators in the cardiovascular protection of salvianolic acid. The interaction with CD36 might also have important implications in plaque cell formation during atherosclerosis.

\section{Salvianolic Acid A}

The beneficial effects of SalA have been studied in the cardiovascular system, including antioxidative, antithrombotic, antifibrotic, anti-inflammatory, and pleotropic effects to protect the myocardium and macro- and microvasculature [55].

4.1. Effects of SalA on Vascular Dysfunction. Endothelial dysfunction is the initiating event leading to vascular inflammation, vascular remodeling, and eventually causing cardiovascular diseases. Therefore, there is a continuous search for new drugs and new drug targets against endothelial dysfunction. Several studies explored the effect of salvianolic acids on endothelial dysfunction using various models. Sun and others $[19,56,57]$ studied the effects of SalA on endothelial dysfunction and vascular remodeling using disease models including angioplasty-related restenosis, injury-induced neointimal hyperplasia, oxidative stress, and advanced glycation end product- (AGE-) induced endothelial dysfunction through enhancing endothelial nitric oxide synthase (eNOS) and its upstream regulators such as cAMP-related signaling. Studies also demonstrated that SalA improves endotheliumdependent vasodilation impaired in both models of spontaneously hypertensive rats [22] and the high-fat, highfructose-induced diabetic rats [57], through enhancing nitric oxide (NO) bioavailability, without affecting blood pressure. SalA can also reverse ischemia/reperfusion-induced reduction in NO bioavailability by reducing MKP-3 (mitogen-activated protein kinase phosphatase 3) in HUVECs [58].

Apart from NO-related pathways, SalA also regulates many other targets which might indirectly modulate NO bioavailability. For instance, SalA inhibits endothelial cell damage induced by AGE through enhancing the antioxidant capacity and the upregulation of eNOS [57]. A recent study showed that SalA is a safe endothelin-1 type A receptor (ETAR) antagonist $(\mathrm{IC} 50=5.7 \mu \mathrm{mol} / \mathrm{L})$ in HEK293 cells overexpressing ETAR [59], suggesting that SalA may be effective in treating hypertension because endothelin-1 is a potent vasoconstrictor. SalA reduces angiotensin II-induced proliferation of human endothelial cell by inhibiting ROS production and blocking phosphorylation of Src and Akt [33]. SalA also attenuates PDGF-BB- (platelet-derived growth factor-BB-) induced VSMC proliferation and migration by inhibiting PDGFR $\beta /$ ERK [56] and the cyclic adenosine monophosphate/protein kinase A/CREB signaling, thus preventing neointimal hyperplasia in rat [19].

In addition to the effect on large vessels, SalA also maintains endothelial homeostasis in microvascular circulation. Teng et al. demonstrated that in SHRs, SalA treatment between 2.5 and $10 \mathrm{mg} / \mathrm{kg} /$ day for 4 weeks attenuates the retinal microvascular inward remodeling and improves the microvascular function of the mesentery in vivo [22]. They also showed that SalA improves endothelial monolayer integrity in vitro, suggesting the therapeutic potential of SalA to prevent organ damage caused by vascular remodeling in the context of hypertension [22]. Yang et al. showed that SalA improves endothelial homeostasis and reduces permeability by modulating tight junction protein $\mathrm{ZO}-1$ and actin cytoskeletal reorganization and reducing ROS in HUVECs exposed to ischemic reperfusion injury. Such effect is mediated through the inhibition or inactivation of p38 MAPK and downregulation of VLDL receptor in endothelial cells [60]. Both studies showed that SalA helps to maintain endothelial homeostasis in microcirculation [22].

4.2. Effects of SalA on Vascular Inflammation. Dysregulated endothelium triggers vascular inflammatory response through interaction with immune cells, among which macrophage is an important player especially during atherogenesis. SalA inhibits lipopolysaccharide- (LPS-) induced upregulation of proinflammatory mediators by targeting IKK $\beta$ (NF$\kappa \mathrm{B}$ kinase inhibitor) and the antioxidative enzyme $\mathrm{HO}-1$, both inhibiting NF- $\kappa \mathrm{B}$ and its target genes including COX2 , iNOS, TNF- $\alpha$, and IL-6 in macrophages [61, 62]. SalA also attenuates angiotensin II-induced macrophage apoptosis by inhibiting Akt and NF- $\kappa \mathrm{B}$ activation, both of which are involved in response to various proinflammatory stimuli [63]. It remains to be investigated whether SalA affects cholesterol uptake and efflux, the subsequent foam cell formation, and efferocytosis of macrophages, which are also important functions of plaque macrophages contributing to the enlargement and resolution of atherosclerotic plaque. Moreover, acetyl-modified SalA inhibits platelet aggregation induced by various proagglutination stimulations, including thrombin, collagen, ADP, and arachidonic acid, suggesting that acetyl-SalA has more potent antithrombotic activity than SalA. Subsequent in vitro and in vivo studies confirmed that SalA inhibits ADP and collagen-induced platelet aggregation and arterial thrombosis in mice [64-67]. These evidences indicated that SalA acts on several vascular cell types through similar signaling molecules including $\mathrm{NF}-\kappa \mathrm{B}$ and $\mathrm{HO}-1$ to inhibit inflammatory responses.

\section{Salvianolic Acid B}

5.1. Effects of SalB on Vascular Dysfunction. Similar to SalA, SalB can also act on the Nrf2-ARE signaling pathway and the p38-MAPK signaling pathway, both of which are closely associated with atherogenesis. Lee et al. showed that SalB inhibits VSMC proliferation and migration induced by PDGFs, accompanied by the upregulation of $\mathrm{HO}-1$ to reduce ROS production [68]. Similarly in HUVECs, SalB upregulates $\mathrm{HO}-1$ and, as a consequence, also inhibits the activation of NF- $\kappa \mathrm{B}$ during the process of cell migration and inflammation induced by TNF- $\alpha$. Further mechanistic studies showed that the upregulation of HO-1 by SalB is Nrf2-dependent. Ling et al. examined the vascular protection of SalB in angiotensin II-induced hypertensive mice. They found that SalB $(25 \mathrm{mg} / \mathrm{kg} /$ day $)$ treatment for 11 days reverses impaired endothelial function and significantly inhibits AT1 receptordependent vascular oxidative stress through inhibition of 
AT1 receptor, as well as NADPH oxidases NOX2 and NOX4 [69]. However, compared to SalA, there have been less studies on the potential benefits of SalB against vascular dysfunction-related diseases.

5.2. Effects of SalB on Vascular Inflammation. SalB can act on various aspects of the interaction between endothelial cells and immune cells to dampen vascular inflammation, which is particularly important at the early stage of atherogenesis. SalB inhibits TNF- $\alpha$ induced endothelial cell-leukocyte adhesion molecule expression on human aortic endothelial cells, in particular the two major adhesion molecules VCAM-1 and ICAM-1, through inhibition of NF- $\kappa$ B activity induced by TNF- $\alpha$, without affecting E-selectin (another major adhesion molecule expressed on the endothelial cells triggered by inflammatory stimuli) [43]. Besides TNF- $\alpha$, SalB also targets interferon-induced response by probably a direct inhibition with IFN-induced phosphorylation of JAK2 (at tyrosine 1007/1008) and also phosphorylation of STAT1 (at tyrosine 701 and serine 727) [70]. This leads to the downregulation of IP-10, a target chemical attractant downstream of STAT1, and suppression of cell adhesion in IFN- $\gamma$-stimulated endothelial cells. In addition, SalB can upregulate PIAS1 and SOCS1, two inhibitory factors of the proinflammatory JAKSTAT1 signaling pathway [70]. Recently, Yang et al. found that SalB can not only inhibit the expression of YAP/TAZ and inflammatory proteins (JNK, NF- $\kappa \mathrm{B}$, and TNF- $\alpha$ ) in $\mathrm{EC}$ and pericytes but also protect $\mathrm{EC}$ and pericytes from oxidative stress and apoptosis. In vivo experiments confirmed that $\mathrm{SalB}$ reduces atherosclerosis in $\mathrm{ApoE}^{-/-}$mice and reduces inflammatory markers including IL- 6 , IL- $1 \beta$, TNF- $\alpha$, and oxLDL in the serum sample of $\mathrm{ApoE}^{-/-}$mice fed a high-fat diet [71].

Platelet-mediated vascular inflammation also contributes to the development and progression of atherosclerosis. A growing body of evidences suggests that SalB may be a potential candidate for the treatment of various atherosclerotic diseases by acting on platelets or endothelial-platelet interaction. For instance, SalB attenuates platelet-triggered inflammation in endothelial cells mediated by NF- $\kappa \mathrm{B}$ activation [71-73]. In platelets, SalB inhibits both ADP and thrombin-induced platelet aggregation by reducing the release of soluble P-selectin and antagonizing the activity of phosphodiesterase and $\mathrm{P} 2 \mathrm{Y} 12$ receptors expressed on the platelets [71-73]. NF- $\kappa \mathrm{B}$ inhibition by SalB is one of the major mechanisms to reduce platelet aggregation and intravascular coagulation in vascular inflammation by SalB acting on both platelets and endothelial cells [72-77].

Effects of SalB on inflammatory response of VSMCs have also been studied. Studies showed that SalB inhibits ERK1/2 and JNK phosphorylation in VSMCs and reduces COX-2mediated production of $\mathrm{PGE}_{2}$, a major inflammatory prostanoid, and NADPH oxidase activity, therefore reducing the proliferation and migration of VSMCs through suppression of MMP-2 and MMP-9, two major enzymes responsible for degradation of the adventitial extracellular matrix, and stimulation of smooth muscle cell migration and proliferation [46, 47]. As a result, in ApoE knockout mice fed with a high-cholesterol diet, supplementation with $0.3 \%$ of SalB for 3 months protects mice from atherosclerosis by reducing vascular intimal thickness, accompanied by decreasing COX2, MMP-2, and MMP-9 expression [46, 47]. In addition, SalB also inhibits TNF- $\alpha$, angiotensin II, or $\mathrm{H}_{2} \mathrm{O}_{2}$-induced MMP2 activation in VSMCs through the inhibition of NADPH oxidase-independent ROS generation [48].

In addition to its effect on endothelial cells against inflammatory responses, SalB also modulates the immune cell population in the plaque. For instance, SalB can retard the maturation of dendritic cells from monocytes accelerated by oxLDL through inhibition of costimulatory molecules CD40 and CD86, mediators of antigen presentation including CD1a and HLA-DR, and proinflammatory and immunomodulatory cytokine IL-12, IL-10, and TNF- $\alpha$ production [78]. Importantly, SalB also activates PPAR $\gamma$ to attenuate oxLDL-induced TLR4 and MyD88 innate immune pathway and its downstream p38-MAPK signaling cascade in dendritic cells [78]. In macrophages, activation of PPAR $\gamma$ along with LXR $\alpha$ by SalB promotes cholesterol efflux through ABCA1 upregulation and reduces LDL uptake by antagonizing CD36-mediated lipid uptake in monocyte-derived macrophages [79], thereby preventing macrophages to become a foam cell $[54,79,80]$. In microglia cells, SalB also reduces the production of NO, TNF- $\alpha$, IL-1b, and ROS induced by LPS through similar mechanisms as those found in endothelial cells [81]. Moreover, SalB might also regulate T lymphocytes, another major player to amplify inflammation in atherogenesis, through inhibition of IL-2, IL-4, TNF- $\alpha$, and IFN- $\gamma$ production, therefore decreasing $\mathrm{T}$ cell activation reflected by inhibition of CD25 and CD69, due to the suppression of transcriptional activity of AP-1, NF- $\kappa \mathrm{B}$, and Oct-1 [82]. The effect of SalB on NF- $\kappa$ B is related to the inhibition of JNK and degradation of $\mathrm{I} \kappa \mathrm{B} \alpha$. These evidences also suggested a potential immunomodulatory effect of SalB, which is different from SalA.

\section{Conclusions and Future Perspectives}

As major bioactive components from Salvia miltiorrhiza (Danshen), there are still many ongoing developments to studying the effect of SalA and SalB on targeting different tissues and various signaling pathways to explore their effect on human diseases, not only restrained to cardiovascular diseases (summarized in Figure 2). Improving the formulation techniques to preserve the bioactive property and stability in vivo is still needed. Extensive evidences have demonstrated the effectiveness of SalA and SalB on vascular dysfunction and vascular inflammation using many animal models to mimic human cardiovascular disease. Both SalA and SalB have effects against oxidative stress, platelet aggregation, coagulation, thrombosis, endothelial dysfunction, and inflammation targeting multiple vascular cell types. As a potent polyphenol, SalA and SalB have direct ROS scavenging ability against different types of free radicals. A more quantitative and reliable method to directly measure scavenging property against free radicals such as superoxide, $\mathrm{H}_{2} \mathrm{O}_{2}$, or biomolecules such as oxLDL in both cell-free and in vivo situation will be extremely informative for future drug development and pharmacokinetic study, for 


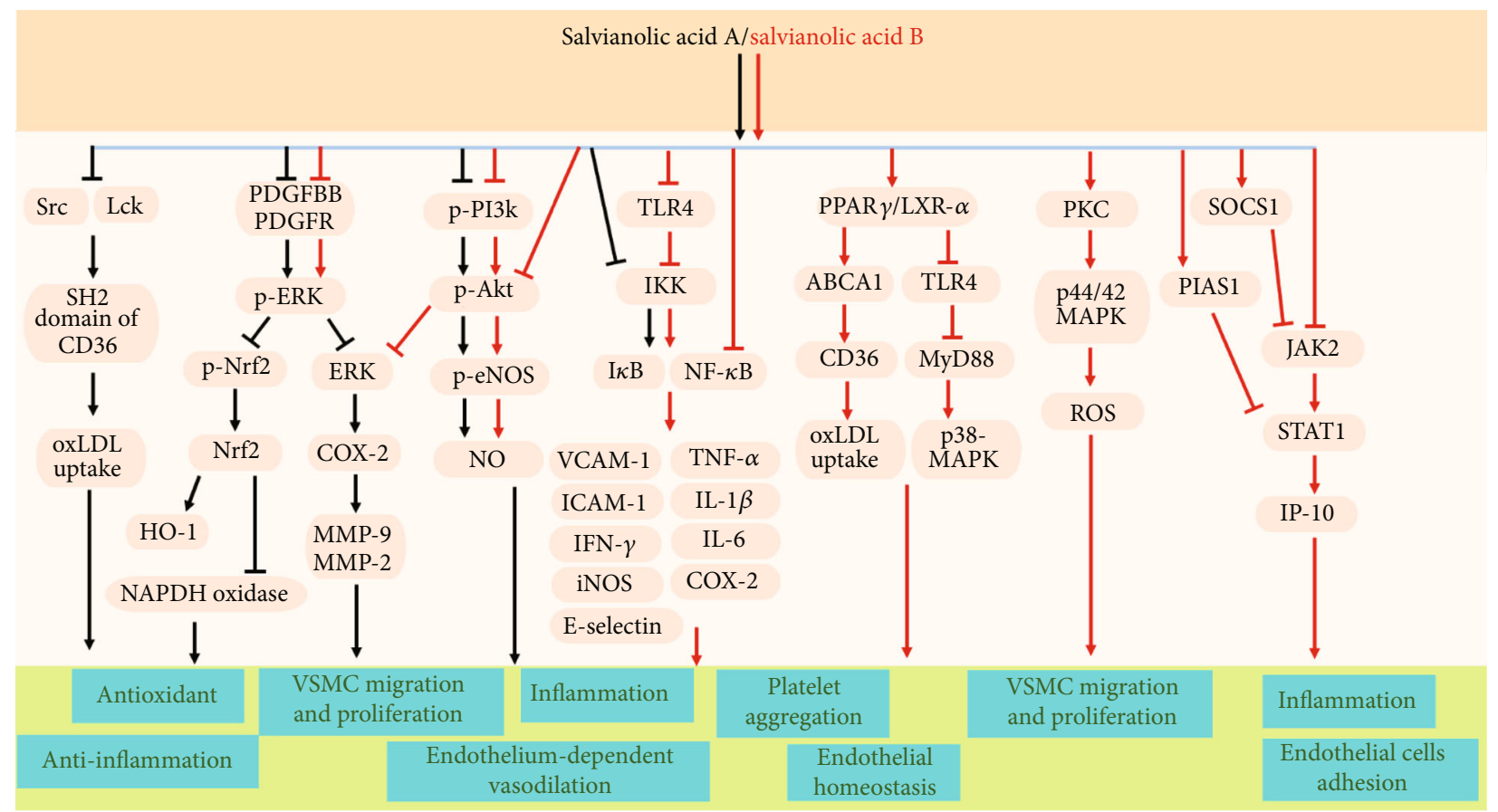

FIGURE 2: The major signaling pathways involved in the vascular protection of salvianolic acid A (SalA) and salvianolic acid B (SalB).

example, using electron spin resonance spin trapping technique. To conclude, the prominent antioxidant, anti-inflammatory, and antifibrotic effects of SalA and SalB translate into the broad cardiovascular actions of Danshen and implicate that both bioactive compounds represent viable drug candidates in cardiovascular disease therapy.

In addition, SalA and SalB might have direct effect to regulate protein phosphorylation on kinases such as ERK, MAPK, and SH2 domain of the Src family kinases. More mechanistic study, for example, using nonbiased proteomics to detect protein phosphorylation treated with SalA and SalB, will provide more information on their interactions with intracellular signaling molecules, in the purpose of detecting new targets. Further animal study using transgenic animals to examine the effect of SalA and SalB in disease models will also be helpful.

\section{Data Availability}

The data used to support the content of this manuscript are available upon request to the corresponding author.

\section{Conflicts of Interest}

The authors declare no conflict of interest.

\section{Authors' Contributions}

All the three authors wrote and reviewed the manuscript.

\section{Acknowledgments}

This study is supported by the NSFC Major Research Programme 91739103 and Young Scientist Fund 81922078 from the National Natural Science Foundation of China, the Early Career Scheme 24122318 and General Research Fund 14109519 from the Hong Kong Research Grant Council, Health and Medical Research Fund 05162906 from the Hong Kong Food and Health Bureau, and start-up funding from the School of Biomedical Sciences, Chinese University of Hong Kong.

\section{References}

[1] World Health Organization, Cardiovascular diseases (CVDs), WHO, 2016, May 15, 2016.

[2] World Health Organization, The top 10 causes of death, WHO, 2017, August 29, 2017.

[3] WHO (World Health Organization), Fact sheet on noncommunicable diseases, B. Ha, S. H. Lee, and D. Park, Eds., 2018, June 2018, https://www.who.int/news-room/fact-sheets/ detail/noncommunicable-diseases.

[4] J. D. Adams, R. Wang, J. Yang, and E. J. Lien, "Preclinical and clinical examinations of Salvia miltiorrhiza and its tanshinones in ischemic conditions," Chinese Medicine, vol. 1, no. 1, p. 3, 2006.

[5] L. Zhou, Z. Zuo, and M. S. Chow, "Danshen: an overview of its chemistry, pharmacology, pharmacokinetics, and clinical use," Journal of Clinical Pharmacology, vol. 45, no. 12, pp. 13451359, 2005.

[6] S. Gao, Z. Liu, H. Li, P. J. Little, P. Liu, and S. Xu, "Cardiovascular actions and therapeutic potential of tanshinone IIA," Atherosclerosis, vol. 220, no. 1, pp. 3-10, 2012. 
[7] Z. Chen and H. Xu, "Anti-inflammatory and immunomodulatory mechanism of tanshinone IIA for atherosclerosis," Evidence-based Complementary and Alternative Medicine, vol. 2014, Article ID 267976, 6 pages, 2014.

[8] S. Xu and P. Liu, "Tanshinone II-A: new perspectives for old remedies," Expert Opinion on Therapeutic Patents, vol. 23, no. 2, pp. 149-153, 2012.

[9] X. Chen, J. Guo, J. Bao, J. Lu, and Y. Wang, "The anticancer properties of Salvia miltiorrhiza Bunge (Danshen): a systematic review," Medicinal Research Reviews, vol. 34, no. 4, pp. 768-794, 2014.

[10] X. Wang, S. L. Morris-Natschke, and K. H. Lee, "New developments in the chemistry and biology of the bioactive constituents of Tanshen," Medicinal Research Reviews, vol. 27, no. 1, pp. 133-148, 2007.

[11] T. Wu, J. Ni, and J. Wu, "Danshen (Chinese medicinal herb) preparations for acute myocardial infarction," Cochrane Database of Systematic Reviews, no. 2, Article ID CD004465, 2008.

[12] T. Y. Yang, J. C. Wei, M. Y. Lee, C. M. Chen, and K. C. Ueng, "A randomized, double-blind, placebo-controlled study to evaluate the efficacy and tolerability of Fufang Danshen (Salvia miltiorrhiza) as add-on antihypertensive therapy in Taiwanese patients with uncontrolled hypertension," Phytotherapy Research, vol. 26, no. 2, pp. 291-298, 2012.

[13] H. C. Zhang, W. Liu, H. T. Yuan, and Y. S. Tang, "Salvia miltiorrhiza reduces plasma levels of asymmetric ADMA in patients with non-ST elevation myocardial infarction undergoing percutaneous coronary intervention," Zhongguo Zhong Xi Yi Jie He Za Zhi, vol. 34, no. 12, pp. 1436-1439, 2014.

[14] D. Zhang, J. Wu, S. Liu, X. Zhang, and B. Zhang, "Salvianolate injection in the treatment of unstable angina pectoris: a systematic review and meta-analysis," Medicine, vol. 95, no. 51, article e5692, 2016.

[15] Q. Zhang, F. Jiao, and C. Hua, "Perioperative application of salvianolate on oxidative stress and plasma IMD/ADM2 in patients with acute myocardial infarction undergoing PCI," Experimental and Therapeutic Medicine, vol. 13, no. 4, pp. 1475-1479, 2017.

[16] C. Grootaert, S. Kamiloglu, E. Capanoglu, and J. Van Camp, "Cell systems to investigate the impact of polyphenols on cardiovascular health," Nutrients, vol. 7, no. 11, pp. 9229-9255, 2015.

[17] O. D. Rangel-Huerta, B. Pastor-Villaescusa, C. M. Aguilera, and A. Gil, "A systematic review of the efficacy of bioactive compounds in cardiovascular disease: phenolic compounds," Nutrients, vol. 7, no. 7, pp. 5177-5216, 2015.

[18] Y. C. Chen, T. Y. Yuan, H. F. Zhang et al., "Salvianolic acid A attenuates vascular remodeling in a pulmonary arterial hypertension rat model," Acta Pharmacologica Sinica, vol. 37, no. 6, pp. 772-782, 2016.

[19] L. Sun, R. Zhao, L. Zhang et al., "Prevention of vascular smooth muscle cell proliferation and injury-induced neointimal hyperplasia by CREB-mediated p21 induction: an insight from a plant polyphenol," Biochemical Pharmacology, vol. 103, pp. 40-52, 2016.

[20] J. Zhang, S. J. An, J. Q. Fu et al., "Mixed aqueous extract of Salvia miltiorrhiza reduces blood pressure through inhibition of vascular remodelling and oxidative stress in spontaneously hypertensive rats," Cellular Physiology and Biochemistry, vol. 40, no. 1-2, pp. 347-360, 2016.
[21] Y. Pan, H. Fu, Q. Kong et al., "Prevention of pulmonary fibrosis with salvianolic acid a by inducing fibroblast cell cycle arrest and promoting apoptosis," Journal of Ethnopharmacology, vol. 155, no. 3, pp. 1589-1596, 2014.

[22] F. Teng, Y. Yin, Y. Cui et al., "Salvianolic acid A inhibits endothelial dysfunction and vascular remodeling in spontaneously hypertensive rats," Life Sciences, vol. 144, pp. 86-93, 2016.

[23] S. A. Jimenez and S. Piera-Velazquez, "Endothelial to mesenchymal transition (EndoMT) in the pathogenesis of systemic sclerosis-associated pulmonary fibrosis and pulmonary arterial hypertension. Myth or reality?," Matrix Biology, vol. 51, pp. 26-36, 2016.

[24] Y. L. Liu and G. T. Liu, "Inhibition of human low-density lipoprotein oxidation by salvianolic acid-A," Yao Xue Xue Bao, vol. 37, no. 2, pp. 81-85, 2002.

[25] Y. Z. Fang, S. Yang, and G. Wu, "Free radicals, antioxidants, and nutrition," Nutrition, vol. 18, no. 10, pp. 872-879, 2002.

[26] G. R. Zhao, H. M. Zhang, T. X. Ye et al., "Characterization of the radical scavenging and antioxidant activities of danshensu and salvianolic acid B," Food and Chemical Toxicology, vol. 46, no. 1, pp. 73-81, 2008.

[27] Y. Sun, H. Zhu, J. Wang, Z. Liu, and J. Bi, "Isolation and purification of salvianolic acid A and salvianolic acid B from Salvia miltiorrhiza by high-speed counter-current chromatography and comparison of their antioxidant activity," Journal of Chromatography. B, Analytical Technologies in the Biomedical and Life Sciences, vol. 877, no. 8-9, pp. 733-737, 2009.

[28] G. T. Liu, T. M. Zhang, B. E. Wang, and Y. W. Wang, "Protective action of seven natural phenolic compounds against peroxidative damage to biomembranes," Biochemical Pharmacology, vol. 43, no. 2, pp. 147-152, 1992.

[29] P. Hu, Q. L. Liang, G. A. Luo, Z. Z. Zhao, and Z. H. Jiang, "Multi-component HPLC fingerprinting of Radix Salviae miltiorrhizae and its LC-MS-MS identification," Chemical and Pharmaceutical Bulletin, vol. 53, no. 6, pp. 677-683, 2005.

[30] Y. J. Wu, C. Y. Hong, S. J. Lin, P. Wu, and M. S. Shiao, "Increase of vitamin E content in LDL and reduction of atherosclerosis in cholesterol-fed rabbits by a water-soluble antioxidant-rich fraction ofSalvia miltiorrhiza," Arteriosclerosis, Thrombosis, and Vascular Biology, vol. 18, no. 3, pp. 481486, 1998.

[31] S. B. Wang, S. Tian, F. Yang, H. G. Yang, X. Y. Yang, and G. H. $\mathrm{Du}$, "Cardioprotective effect of salvianolic acid A on isoproterenol-induced myocardial infarction in rats," European Journal of Pharmacology, vol. 615, no. 1-3, pp. 125-132, 2009.

[32] B. Liu, B. Cao, D. Zhang et al., "Salvianolic acid B protects against paraquat-induced pulmonary injury by mediating Nrf2/Nox4 redox balance and TGF- $\beta 1 /$ Smad3 signaling," Toxicology and Applied Pharmacology, vol. 309, pp. 111-120, 2016.

[33] L. L. Yang, D. Y. Li, Y. B. Zhang, M. Y. Zhu, D. Chen, and T. D. $\mathrm{Xu}$, "Salvianolic acid A inhibits angiotensin II-induced proliferation of human umbilical vein endothelial cells by attenuating the production of ROS," Acta Pharmacologica Sinica, vol. 33, no. 1, pp. 41-48, 2012.

[34] H. Tang, C. S. Pan, X. W. Mao et al., "Role of NADPH oxidase in total salvianolic acid injection attenuating ischemiareperfusion impaired cerebral microcirculation and neurons: implication of AMPK/Akt/PKC," Microcirculation, vol. 21, no. 7, pp. 615-627, 2014. 
[35] M. K. Tsai, Y. L. Lin, and Y. T. Huang, "Effects of salvianolic acids on oxidative stress and hepatic fibrosis in rats," Toxicology and Applied Pharmacology, vol. 242, no. 2, pp. 155-164, 2010.

[36] Y. Shu, Y. Liu, X. Li et al., "Aspirin-triggered resolvin D1 inhibits TGF- $\beta 1$-induced EndMT through increasing the expression of Smad7 and is closely related to oxidative stress," Biomolecules \& Therapeutics, vol. 24, no. 2, pp. 132-139, 2016.

[37] Y. Chen, T. Yuan, H. Zhang et al., "Activation of Nrf2 attenuates pulmonary vascular remodeling via inhibiting endothelial-to-mesenchymal transition: an insight from a plant polyphenol," International Journal of Biological Sciences, vol. 13, no. 8, pp. 1067-1081, 2017.

[38] Y. C. Hung, P. W. Wang, T. L. Pan, G. Bazylak, and Y. L. Leu, "Proteomic screening of antioxidant effects exhibited by radix Salvia miltiorrhiza aqueous extract in cultured rat aortic smooth muscle cells under homocysteine treatment," Journal of Ethnopharmacology, vol. 124, no. 3, pp. 463-474, 2009.

[39] T. J. Lin, G. T. Liu, Y. Liu, and G. Z. Xu, "Protection by salvianolic acid A against adriamycin toxicity on rat heart mitochondria," Free Radical Biology \& Medicine, vol. 12, no. 5, pp. 347-351, 1992.

[40] B. Jiang, L. Zhang, M. Li et al., "Salvianolic acids prevent acute doxorubicin cardiotoxicity in mice through suppression of oxidative stress," Food and Chemical Toxicology, vol. 46, no. 5, pp. 1510-1515, 2008.

[41] S. Liu, Y. Sun, H. Chen, S. Song, and Y. Xu, "Magnetic screening of the potential targeted protein of salvianolic acid B using T7 phage display library," Applied Biochemistry and Biotechnology, vol. 162, no. 4, pp. 1206-1213, 2010.

[42] B. Jiang, J. Chen, L. Xu et al., "Salvianolic acid B functioned as a competitive inhibitor of matrix metalloproteinase- 9 and efficiently prevented cardiac remodeling," BMC Pharmacology, vol. 10, no. 1, p. 10, 2010.

[43] Y. H. Chen, S. J. Lin, H. H. Ku et al., "Salvianolic acid B attenuates VCAM-1 and ICAM-1 expression in TNF-alpha-treated human aortic endothelial cells," Journal of Cellular Biochemistry, vol. 82, no. 3, pp. 512-521, 2001.

[44] M. Ding, T. X. Ye, G. R. Zhao, Y. J. Yuan, and Z. X. Guo, "Aqueous extract of Salvia miltiorrhiza attenuates increased endothelial permeability induced by tumor necrosis factoralpha," International Immunopharmacology, vol. 5, no. 11, pp. 1641-1651, 2005.

[45] Z. Zhou, Y. Liu, A. D. Miao, and S. Q. Wang, "Salvianolic acid $\mathrm{B}$ attenuates plasminogen activator inhibitor type 1 production in TNF-alpha treated human umbilical vein endothelial cells," Journal of Cellular Biochemistry, vol. 96, no. 1, pp. 109-116, 2005.

[46] Y. L. Chen, C. S. Hu, F. Y. Lin et al., "Salvianolic acid B attenuates cyclooxygenase-2 expression in vitro in LPS-treated human aortic smooth muscle cells and in vivo in the apolipoprotein-E-deficient mouse aorta," Journal of Cellular Biochemistry, vol. 98, no. 3, pp. 618-631, 2006.

[47] S. J. Lin, I. T. Lee, Y. H. Chen et al., "Salvianolic acid B attenuates MMP-2 and MMP-9 expression in vivo in apolipoprotein-E-deficient mouse aorta and in vitro in LPStreated human aortic smooth muscle cells," Journal of Cellular Biochemistry, vol. 100, no. 2, pp. 372-384, 2007.

[48] H. S. Zhang and S. Q. Wang, "Salvianolic acid B from Salvia miltiorrhiza inhibits tumor necrosis factor-alpha (TNFalpha)-induced MMP-2 upregulation in human aortic smooth muscle cells via suppression of $\mathrm{NAD}(\mathrm{P}) \mathrm{H}$ oxidase-derived reactive oxygen species," Journal of Molecular and Cellular Cardiology, vol. 41, no. 1, pp. 138-148, 2006.

[49] J. Schlessinger and M. A. Lemmon, "SH2 and PTB domains in tyrosine kinase signaling," Science's STKE, vol. 2003, no. 191, p. RE12, 2003.

[50] M. B. Yaffe and S. J. Smerdon, "The use of in vitro peptidelibrary screens in the analysis of phosphoserine/threoninebinding domain structure and function," Annual Review of Biophysics and Biomolecular Structure, vol. 33, no. 1, pp. 225-244, 2004.

[51] B. Sperl, M. H. Seifert, and T. Berg, "Natural product inhibitors of protein-protein interactions mediated by Src-family $\mathrm{SH} 2$ domains," Bioorganic \& Medicinal Chemistry Letters, vol. 19, no. 12, pp. 3305-3309, 2009.

[52] J. Won, Y. G. Hur, E. M. Hur et al., "Rosmarinic acid inhibits TCR-induced $\mathrm{T}$ cell activation and proliferation in an Lckdependent manner," European Journal of Immunology, vol. 33, no. 4, pp. 870-879, 2003.

[53] W. C. Shakespeare, "SH2 domain inhibition: a problem solved?," Current Opinion in Chemical Biology, vol. 5, no. 4, pp. 409-415, 2001.

[54] L. Wang, Y. Bao, Y. Yang et al., "Discovery of antagonists for human scavenger receptor CD36 via an ELISA-like highthroughput screening assay," Journal of Biomolecular Screening, vol. 15, no. 3, pp. 239-250, 2010.

[55] J. H. Ho and C. Y. Hong, "Salvianolic acids: small compounds with multiple mechanisms for cardiovascular protection," Journal of Biomedical Science, vol. 18, no. 1, p. 30, 2011.

[56] L. Sun, R. Zhao, L. Zhang et al., "Salvianolic acid A inhibits PDGF-BB induced vascular smooth muscle cell migration and proliferation while does not constrain endothelial cell proliferation and nitric oxide biosynthesis," Molecules, vol. 17, no. 3, pp. 3333-3347, 2012.

[57] X. Y. Yang, G. F. Qiang, L. Zhang et al., "Salvianolic acid A protects against vascular endothelial dysfunction in high-fat diet fed and streptozotocin-induced diabetic rats," Journal of Asian Natural Products Research, vol. 13, no. 10, pp. 884894, 2011.

[58] D. Yang, P. Xie, and Z. Liu, "Ischemia/reperfusion-induced MKP-3 impairs endothelial NO formation via inactivation of ERK1/2 pathway," PLoS One, vol. 7, no. 7, article e42076, 2012.

[59] Q. Zhang, S. Wang, Y. Yu et al., "Salvianolic acid A, as a novel ETA receptor antagonist, shows inhibitory effects on tumor in vitro," International Journal of Molecular Sciences, vol. 17, no. 8, p. 1244, 2016.

[60] D. Yang, P. Zhang, T. Wang et al., "SalA attenuates ischemia/reperfusion-induced endothelial barrier dysfunction via downregulation of VLDL receptor expression," Cellular Physiology and Biochemistry, vol. 33, no. 3, pp. 747-757, 2014.

[61] K. S. Oh, B. K. Oh, J. Mun, H. W. Seo, and B. H. Lee, "Salvianolic acid A suppress lipopolysaccharide-induced NF- $\kappa \mathrm{B}$ signaling pathway by targeting IKK $\beta$," International Immunopharmacology, vol. 11, no. 11, pp. 1901-1906, 2011.

[62] J. Huang, Y. Qin, B. Liu, G. Y. Li, L. Ouyang, and J. H. Wang, "In silico analysis and experimental validation of molecular mechanisms of salvianolic acid A-inhibited LPS-stimulated inflammation, in RAW264.7 macrophages," Cell Proliferation, vol. 46, no. 5, pp. 595-605, 2013.

[63] L. Li, T. Xu, Y. Du et al., "Salvianolic acid a attenuates cell apoptosis, oxidative stress, Akt and NF- $\kappa \mathrm{B}$ activation in angiotensin-II induced murine peritoneal macrophages," 
Current Pharmaceutical Biotechnology, vol. 17, no. 3, pp. 283290, 2016.

[64] M. K. Tang, D. C. Ren, J. T. Zhang, and G. H. Du, "Effect of salvianolic acids from Radix Salviae miltiorrhizae on regional cerebral blood flow and platelet aggregation in rats," Phytomedicine, vol. 9, no. 5, pp. 405-409, 2002.

[65] Y. Yao, W. Y. Wu, A. H. Liu et al., "Interaction of salvianolic acids and notoginsengnosides in inhibition of ADP-induced platelet aggregation," The American Journal of Chinese Medicine, vol. 36, no. 2, pp. 313-328, 2012.

[66] Z. S. Huang, C. L. Zeng, L. J. Zhu, L. Jiang, N. Li, and H. Hu, "Salvianolic acid A inhibits platelet activation and arterial thrombosis via inhibition of phosphoinositide 3-kinase," Journal of Thrombosis and Haemostasis, vol. 8, no. 6, pp. 13831393, 2010.

[67] H. Y. Fan, F. H. Fu, M. Y. Yang, H. Xu, A. H. Zhang, and K. Liu, "Antiplatelet and antithrombotic activities of salvianolic acid A," Thrombosis Research, vol. 126, no. 1, pp. e17-e22, 2010.

[68] H. J. Lee, MiRanSeo, and E. J. Lee, "Salvianolic acid B inhibits atherogenesis of vascular cells through induction of Nrf2dependent heme oxygenase-1," Current Medicinal Chemistry, vol. 21, no. 26, pp. 3095-3106, 2014.

[69] W. C. Ling, J. Liu, C. W. Lau, D. D. Murugan, M. R. Mustafa, and Y. Huang, "Treatment with salvianolic acid B restores endothelial function in angiotensin II-induced hypertensive mice," Biochemical Pharmacology, vol. 136, pp. 76-85, 2017.

[70] J. Ba, H. Peng, Y. Chen, and Y. Gao, "Effects and mechanism analysis of vascular endothelial growth factor and salvianolic acid B on 125I-low density lipoprotein permeability of the rabbit aortary endothelial cells," Cell Biochemistry and Biophysics, vol. 70, no. 3, pp. 1533-1538, 2014.

[71] C. Stumpf, Q. Fan, C. Hintermann et al., “Anti-inflammatory effects of Danshenon human vascular endothelial cells in culture," The American Journal of Chinese Medicine, vol. 41, no. 5, pp. 1065-1077, 2013.

[72] S. Xu, A. Zhong, X. Bu et al., "Salvianolic acid B inhibits platelets-mediated inflammatory response in vascular endothelial cells," Thrombosis Research, vol. 135, no. 1, pp. 137145, 2015.

[73] L. Liu, J. Li, Y. Zhang et al., "Salvianolic acid B inhibits platelets as a P2Y12 antagonist and PDE inhibitor: evidence from clinic to laboratory," Thrombosis Research, vol. 134, no. 4, pp. 866876, 2014.

[74] Z. Wu, J. N. Li, Z. Q. Bai, and X. Lin, “Antagonism by salvianolic acid B of lipopolysaccharide-induced disseminated intravascular coagulation in rabbits," Clinical and Experimental Pharmacology \& Physiology, vol. 41, no. 7, pp. 502-508, 2014.

[75] Y. P. Wu, X. M. Zhao, S. D. Pan et al., "Salvianolic acid B inhibits platelet adhesion under conditions of flow by a mechanism involving the collagen receptor alpha2beta1," Thrombosis Research, vol. 123, no. 2, pp. 298-305, 2008.

[76] C. Ma, Y. Yao, Q. X. Yue et al., "Differential proteomic analysis of platelets suggested possible signal cascades network in platelets treated with salvianolic acid B," PLoS One, vol. 6, no. 2, article e14692, 2011.

[77] M. Li, C. Zhao, R. N. Wong, S. Goto, Z. Wang, and F. Liao, "Inhibition of shear-induced platelet aggregation in rat by tetramethylpyrazine and salvianolic acid B," Clinical Hemorheology and Microcirculation, vol. 31, no. 2, pp. 97-103, 2004.
[78] A. Sun, H. Liu, S. Wang et al., "Salvianolic acid B suppresses maturation of human monocyte-derived dendritic cells by activating PPAR $\gamma$," British Journal of Pharmacology, vol. 164, no. 8, pp. 2042-2053, 2011.

[79] J. Yue, B. Li, Q. Jing, and Q. Guan, "Salvianolic acid B accelerated ABCA1-dependent cholesterol efflux by targeting PPAR$\gamma$ and LXR $\alpha$," Biochemical and Biophysical Research Communications, vol. 462, no. 3, pp. 233-238, 2015.

[80] Y. Bao, L. Wang, Y. Xu et al., "Salvianolic acid B inhibits macrophage uptake of modified low density lipoprotein (mLDL) in a scavenger receptor CD36-dependent manner," Atherosclerosis, vol. 223, no. 1, pp. 152-159, 2012.

[81] S. X. Wang, L. M. Hu, X. M. Gao, H. Guo, and G. W. Fan, "Anti-inflammatory activity of salvianolic acid B in microglia contributes to its neuroprotective effect," Neurochemical Research, vol. 35, no. 7, pp. 1029-1037, 2010.

[82] C.-C. Cheng, S.-P. Yang, W.-S. Lin et al., "Magnesium lithospermate $\mathrm{B}$ mediates anti-inflammation targeting activator protein-1 and nuclear factor-kappa B signaling pathways in human peripheral T lymphocytes," International Immunopharmacology, vol. 13, no. 3, pp. 354-361, 2012. 